\title{
Preface
}

\section{The Development of Positive Airway Pressure Technology and Applications: Faster than Space Travel!}

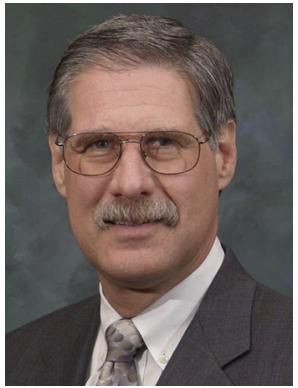

Lee K. Brown, MD

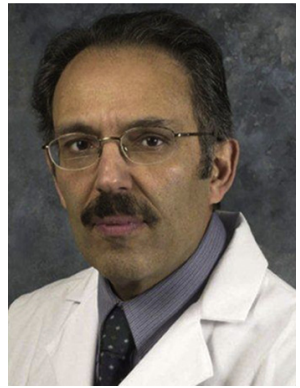

Shahrokh Javaheri, MD

Editors

The editorial focus of this issue of Sleep Medicine Clinics is to describe the rapid evolution of positive airway pressure (PAP) technology and the diverse applications of currently available devices. We are increasingly astounded by the capabilities that have been implemented since the vacuum cleaner blower and custom-fitted interface, first described by Sullivan in 1981, to the current availability of sophisticated devices having multiple operational modes. As an intellectual exercise, it is interesting to compare PAP development to that of spacecraft computational technology over a similar period. Contemporaneous with the origins of CPAP, the space shuttle program began with flight of Columbia on April 12, 1981 and ended in 2011. The space shuttles used, even after an upgrade, IBM AP-101S CPUs that could address only 256 kilobytes of memory and perform an underwhelming 1 million instructions per second (MIPS). The main computers equipping the American section of the International Space Station (ISS), still in active use, employ Intel 80386SX microprocessors. These chips were introduced in 1985, and when clocked at $33 \mathrm{MHz}$, could achieve about 11.4 MIPS and address up to 15 megabytes of memory; not bad for its time, but now sadly out of date. In comparison, beginning around the year 2000, Texas Instruments and other semiconductor manufacturers began marketing a series of CPUs (described as digital processing units in some literature) that could achieve performance far above that in the ISS. For instance, the Texas Instruments TMS320C2000 series that was marketed for use in CPAP generators starting around the year 2000 could run at clock speeds up to $300 \mathrm{MHz}$, perform up to 150 MIPS, had on-chip random access memory and flash (nonvolatile) memory of as much as 516 and 512 kilobytes, respectively (not both maxima in the same chip), along with on-chip functionalities that included analog-to-digital converters, pulse width modulation output for blower motor control, and an external memory bus to access additional memory. And that was 15 years ago and at a cost of no more than $\$ 20$ each in quantity.

The exponential expansion of the functionality of CPUs available for commercial applications has been breathtaking to behold (see Moore's law). In parallel with this expansion, manufacturers of PAP flow generators have rapidly adapted to the strength of computing power now available in tiny form factors and at low prices in order to design flow generators with powerful capabilities that could not have been imagined by Sullivan in 1981. Yet, with great power comes great responsibility. The responsibility undertaken by physicians who prescribe these devices for the use of patients in their homes requires that they be mindful of the 
possibility that such complex devices may not behave as predicted when faced with the myriad patterns of sleep-disordered breathing that can occur in actual patients in the actual home setting. In this issue of Sleep Medicine Clinics, we have endeavored to comprehensively review PAP treatment for sleep-disordered breathing. We have included herein articles by leading experts in the field describing the technology employed by these devices, the choice of flow generator modality and patient interface, techniques for gaining patient acceptance of such treatment, and the nuances associated with the use of the current panoply of PAP generators in patients with a wide variety of conditions whose only similarity is that they exhibit some type (or many types) of sleep-disordered breathing. Our aim is thus to extend the Latin phrase caveat emptor ("let the buyer beware") to more specifically apply to the physician: caveat medicus.

Financial Disclosures: Dr Brown has participated in advisory panels for Philips Respironics and is an insurance claims reviewer for Considine and Associates, Inc. He co-edits the sleep and respiratory neurobiology section of Current Opinion in Pulmonary Medicine, wrote on CPAP treatment for obstructive sleep apnea in UpToDate and on obstructive sleep apnea in Clinical
Decision Support: Pulmonary Medicine and Sleep Disorders. He is currently co-editing an issue of Sleep Medicine Clinics on positive airway pressure therapy. He serves on the Polysomnography Practice Advisory Committee of the New Mexico Medical Board and chairs the New Mexico Respiratory Care Advisory Board. Dr Javaheri has no relevant conflicts of interest.

Lee K. Brown, MD

Department of Internal Medicine

School of Medicine

University of New Mexico

Sleep Disorders Center

1101 Medical Arts Avenue Northeast, Building \#2 Albuquerque, NM 87102, USA

Shahrokh Javaheri, MD

University of Cincinnati

Ohio University Medical School

Sleep Laboratory

Bethesda North Hospital

10475 Montgomery Road

Cincinnati, OH 45242, USA

E-mail addresses:

Ikbrown@alum.mit.edu (L.K. Brown) shahrokhjavaheri@icloud.com (S. Javaheri) 